\title{
On-line thermography applied to crack detection in steel billets.
}

\author{
by J. Wullink $\left(^{*}\right)$ and Ph. Darses $\left(^{* *}\right)$
}

(*) Corus Research, Development \& Technology, PO Box 10.000, 1970 CA IJmuiden, The Netherlands. e-mail: johan.wullink@corusgroup.com

$\left.{ }^{* *}\right)$ CEDIP Infrared Systems, 19 Bd Georges Bidault, ZI Paris EST, F-77183 Croissy-Beaubourg, France. e-mail: philippe.darses@wanadoo.fr

\author{
Abstract \\ indicating the presence of a crack. \\ and results.

\section{Nomenclature} \\ $M_{e}(\lambda, T) \quad$ spectral radiant exitance $\left(\mathrm{W} / \mathrm{m}^{2} / \mathrm{m}\right)$ \\ $\varepsilon(\lambda) \quad$ emissivity (-) \\ $c_{1} \quad$ Planck's first constant $\left(\mathrm{Wm}^{2}\right)$ \\ $c_{2} \quad$ Planck's second constant $(\mathrm{mK})$ \\ $\lambda \quad$ wavelength $(\mathrm{m})$ \\ $T \quad$ temperature of object $(\mathrm{K})$ \\ $\mathrm{a}(\lambda) \quad$ absorption coefficient $\left(\mathrm{cm}^{-1}\right)$ \\ $\alpha(\lambda) \quad$ absorptivity (-) \\ $\tau(\lambda) \quad$ transmission $(-)$ \\ $\mathrm{x} \quad$ thickness of water layer $(\mathrm{cm})$
}

On-line detection of longitudinal cracks in steel billets and bars is performed by inductive heating and infrared scanning of the surface under inspection. In collaboration with CEDIP, CORUS Research, Development \& Technology designed an inspection system based on four infrared matrix cameras. The infrared images are analysed on-line by computer, searching for transverse temperature gradients,

This paper describes the construction of the inspection system, functioning of the crack detection tools

\section{Introduction}

On-line inspection of products is a key issue in modern steel works. It generates quality figures to support process optimization (feedback) and it shields customers from inferior material (feedforward). The presence of longitudinal cracks in steel billets is of great concern for further processing of automotive parts at forging shops. The inspection system introduced named IRUS is capable of detecting these cracks on-line. As the name of the inspection system indicates, an ultra sonic inspection is also integrated, but will not be considered in this paper. 


\section{Hardware set-up and principle of IRUS}

The hardware (figure 1) of IRUS consists of 3 main parts:

- the moistening unit sprays a thin water film over the billet surface in order to increase and equalize its emissivity.

- the inductive heating unit makes the temperature of the surface of the steel billet increase. Because of the high frequency, only a thin layer of $0.2 \mathrm{~mm}$ of the surface is heated by the electric current. In the vicinity of longitudinal cracks local electrical resistance is higher compared to non defect locations, resulting in a higher temperature increase. These locally increased temperatures are detected by the detection unit.

- the detection unit is built up from 4 infrared matrix cameras, mounted directly behind the HF coil and covering the whole circumference of the billet. The billet is analysed by means of a 2D Focal Plane Array infrared camera with $128^{2}$ pixels and a maximum sampling frequency of $50 \mathrm{~Hz}$. The resulting square image covers the range of billet dimensions from $45 \mathrm{~mm}$ up to $125 \mathrm{~mm}$. Because the lens of the camera is fixed and the distance between the camera and the billet surface may vary from one billet to another, a dedicated lens is mounted on the camera with a large focal depth. Furthermore, the lens is covered with a carbon coating to shield from industrial environment.

\section{principle of IRUS billet inspection system.}

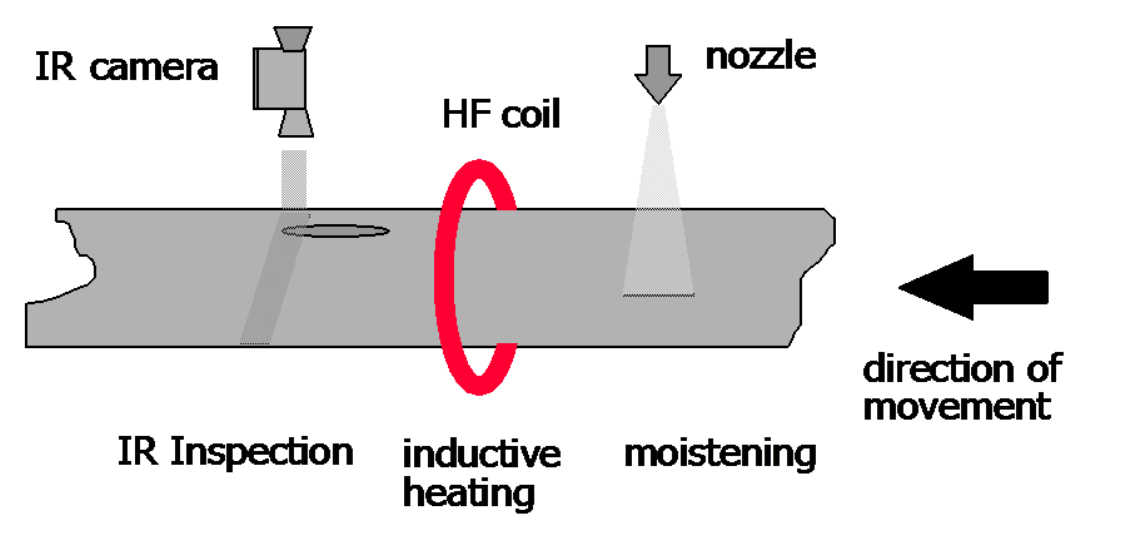

Figure 1: principle of the IRUS billet inspection system.

\section{General considerations and theory}

An important consideration for the IR camera configuration is the choice of the wavelength. In the recent past a large number of documents on this subject have been published. The choice for the wavelength of the camera is practically limited to the 3-5 $\mu \mathrm{m}$ and the 8-12 $\mu \mathrm{m}$ range. We have taken three criteria for choosing the wavelength in consideration:

1. object emissivity vs. wavelength.

2. thermal sensitivity vs. wavelength.

3. instrument parameters related to wavelength. 


\section{Ad.1: object emissivity vs. wavelength}

In the wavelength range of 8 to $12 \mu \mathrm{m}$, the absorption coefficient $\mathrm{a}(\lambda)$ of water has a high and constant value of approximately $1000 \mathrm{~cm}^{-1}$ and a strongly fluctuating, but comparable average value in the 3-5 $\mu \mathrm{m}$ range [1]. The transmission of the water film on the billet surface is expressed by:

$$
\tau=e^{-a x} \quad[-]
$$

for an estimated water film thickness of $x=20 \mu \mathrm{m}, \tau=0.135$.

By Kirchhoff's law:

$$
\alpha(\lambda)=\varepsilon(\lambda) \quad[-]
$$

Neglecting reflectivity, the emissivity of the water film is 0.87 . This value is significantly higher than the value for shotblasted steel surfaces without a water film, for which it is approximately 0.6 .

A drawback of the longer wavelength is the higher sensitivity to emissivity variations of the systems output, according to (for small changes):

$$
\Delta T=\frac{-\lambda T^{2}}{c_{2} \varepsilon} \Delta \varepsilon \quad[\mathrm{K}]
$$

Consequently the shortest possible wavelength would be favourable.

\section{Ad 2: thermal sensitivity vs. wavelength.}

A high sensitivity of radiant exitance to temperature is favourable with regard to a good signal to noise ratio. According to Planck's law, the sensitivity of exitance to temperature is expressed by the first partial derivative of $\mathrm{M}_{\mathrm{e}}(\lambda, \mathrm{T})$ with respect to $\mathrm{T}$ [2]:

$$
\frac{\partial M_{e}(\lambda, T)}{\partial T}=M_{e}(\lambda, T) \frac{c_{2} e^{c_{2} / \lambda T}}{\lambda T^{2}\left[e^{c_{2} / \lambda T}-1\right]} \quad[\mathrm{W} / \mathrm{m} 2 / \mathrm{m} / \mathrm{K}]
$$

As depicted in figure 5, (4) has a maximum at a wavelength of $8 \mu \mathrm{m}$ around $300 \mathrm{~K}$, roughly within the range of the billet temperature after inductive heating. This means that for the highest sensitivity of exitance to temperature, a wavelength of $8 \mu \mathrm{m}$ is favourable.

\section{Ad 3: instrument parameters related to wavelength.}

Because defect detection is executed by means of image analysis, it is essential that the detection system has a sharp infrared image of the surface under inspection. This means that the infrared camera should have the shortest possible integration time. Among the available infrared cameras, the one in the range from 8 to $12 \mu \mathrm{m}$ has a shorter integration time compared to the one in the 3 to $5 \mu \mathrm{m}$ range, i.c. $100 \mu \mathrm{s}$ and $1 \mathrm{~ms}$ respectively. At a maximum speed of $1 \mathrm{~m} / \mathrm{s}$ of the surface under inspection, the displacement equals to $0.1 \mathrm{~mm}$ during the build-up of the infrared image in case of 8 to $12 \mu \mathrm{m}$ wavelength range. This was considered as an acceptable value compared to the pixel resolution of $1.25 \mathrm{~mm}$. 
Considering the three forementioned criteria it was concluded that a short integration time has the highest priority and therefore an infrared camera in the 8 to $12 \mu \mathrm{m}$ range was choosen.

\section{Crack detection procedure}

The choice of a 2D matrix camera in stead of a 1D line camera was made to be more flexible in terms of crack detection strategy. A 2D camera offers the opportunity to spot a crack more than once within the field of view and can make use of the dynamic behaviour of a crack: immediately behind the coil maximum contrast is obtained and within a second it has disappeared.

The crack detection procedure is a multiple pass algorithm based on the following steps :

\subsection{Edge detection \& Region of Interest}

Due to the variable dimension of the billet in the field of view of the camera and the lateral motion of the billet itself due to the conveyor, the system has to search for the position of the billet and track it. An edge detection module is dedicated to this task and is based on a filtering algorithm.

The priciple of this algorithm is to detect a significant contrast on both edges (left and right) of the billet. The contrast between the billet and its surroundings is enhanced by means of the heating of the billet surface by the coil. The following typical image (figure 2) illustrates the response of the camera to a billet in its field of view :
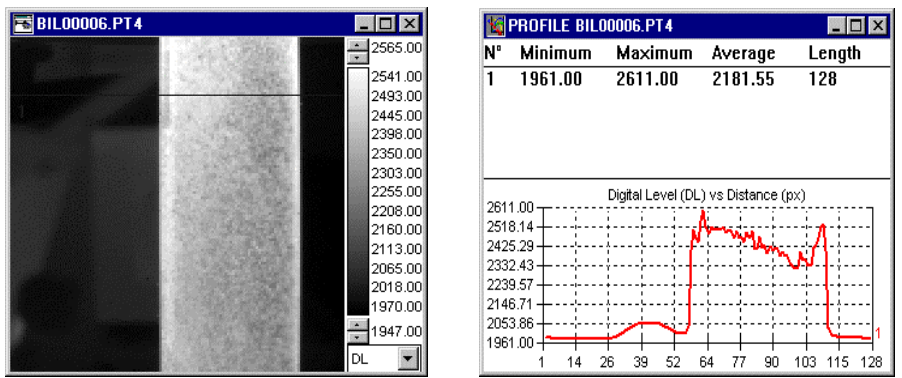

Figure 2: Image \& Line profile of edges

After detection of the billet edge, a constant part of the length of the billet in terms of rows in the matrix is defined, resulting in a rectangle. This rectangle is called the region of interest (figure 3). Synchronously to the speed of the billet, the frame rate of the camera is adapted in such a way, that in every new image of the camera, the displacement of the billet is equal to the length of the region of interest. Every region of interest is analysed on-line by computer.

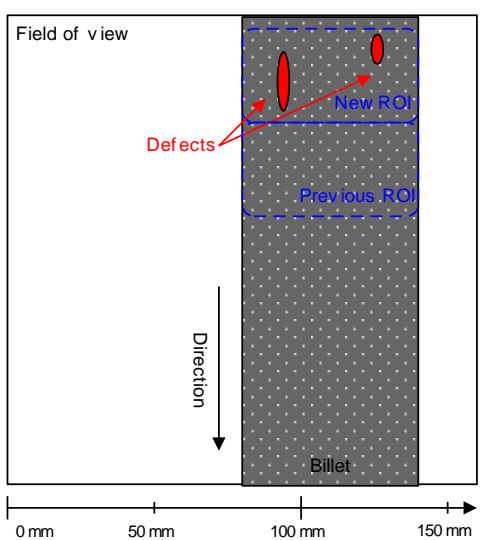

Figure 3 : Region of Interest

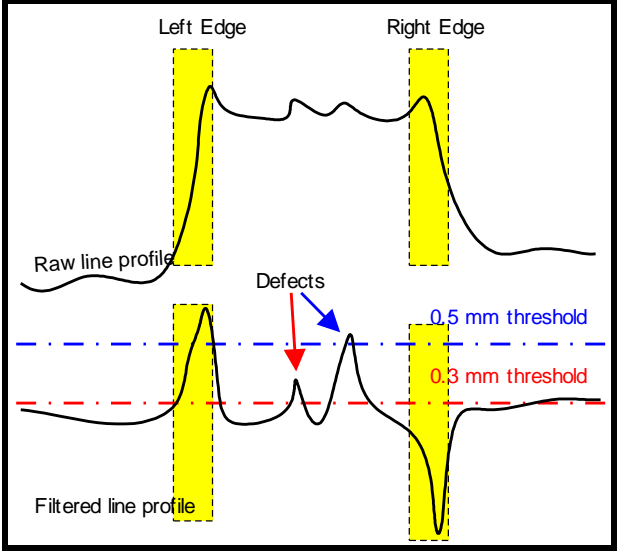

Figure 4 : Filtering \& Threshold 


\subsection{High-Pass Filtering \& Threshold}

A high pass filtering module removes the low frequency from the image. This filter is from a 1D convolution matrix filter. Figure 4 shows the effect of the filtering module on the raw data of one row.

Depending on the target depth of cracks to be detected, a threshold is applied to the data. The data above the threshold are considered as corresponding to a potential crack. After analysing all the rows in the region of interest, the shape of a potential crack is known to the system. The potential crack data have to pass a shape criterium that checks the length/width ratio before it is considered as a real crack. A painting device, located a few metres behind the inspection system sprays a paint track onto the location of the defect. The defect detection procedure is executed for all 4 sides of the billet at the same time.

\subsection{Database}

After the complete inspection of the billet surface, a datasheet is generated with the list of cracks and their $x$ - and $y$ positions on the billet, supplemented by some statistical data including length, width and depth per defect.

\section{Results}

The inspection results of one complete billet can be displayed by a tool called BIRD. (figure 6) The upper part displays an infrared image of one side of a billet, here 8.5 metres long and 70 $\mathrm{mm}$ wide. The lower part has zoomed-in on $20 \mathrm{~cm}$ of length, while the separate regions of interest can be distinguished. Defects are marked by a colored rectangle and are listed in a table in the right bottom corner of the screen.

\section{Conclusion}

In conclusion we can say that an inspection system based on inductive heating and thermal imaging is well capable of on-line detecting longitudinal cracks of depths $\geq 0.3 \mathrm{~mm}$ and lengths $\geq 5 \mathrm{~mm}$ in steel billets.

\section{Prospects}

The use of a 2-D infrared camera offers possibilities for more advanced crack detection, in order to find other types- or shallower defects. This is considered as a challenging research opportunity.

Recent developments in detector technology offer chances to use uncooled matrix detectors which results in less maintenance effort for current cooling devices.

\section{REFERENCES}

[1] Wolfe, W.L. - Introduction to infrared system design. SPIE- The international Society for Optical Engineering, 1996, p 55.

[2] HOLST, G.C. - Testing and evaluation of infrared imaging systems. JCD Publishing, 1993, p. 73 - 78. 


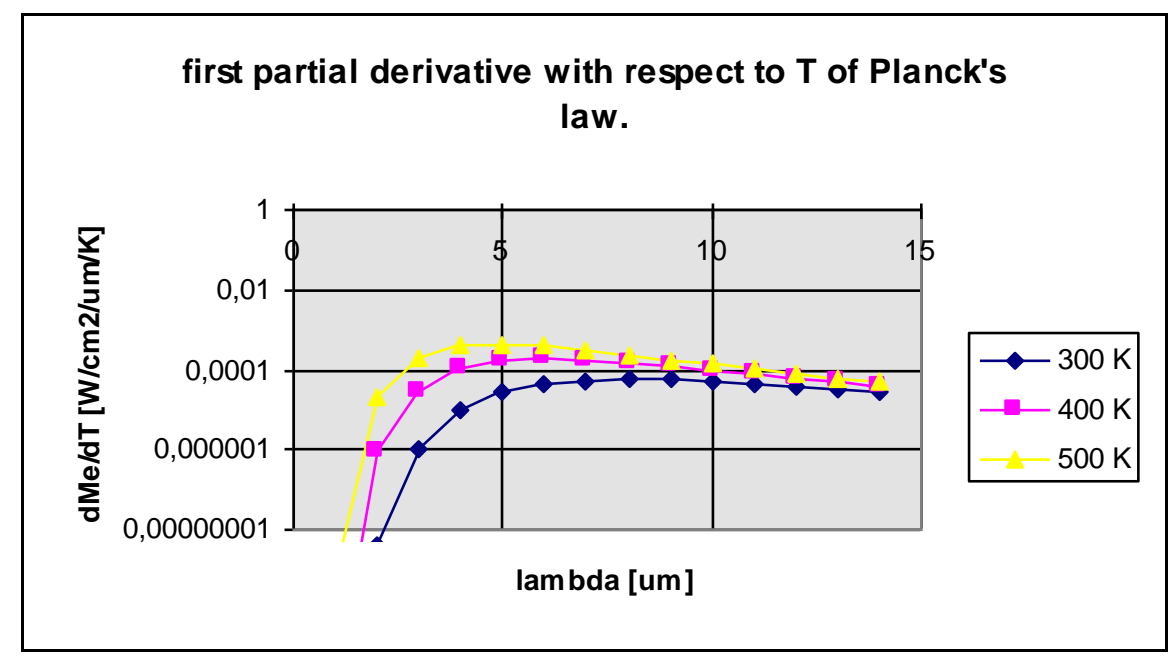

figure 5 : First partial derivative with respect to T of Planck's law

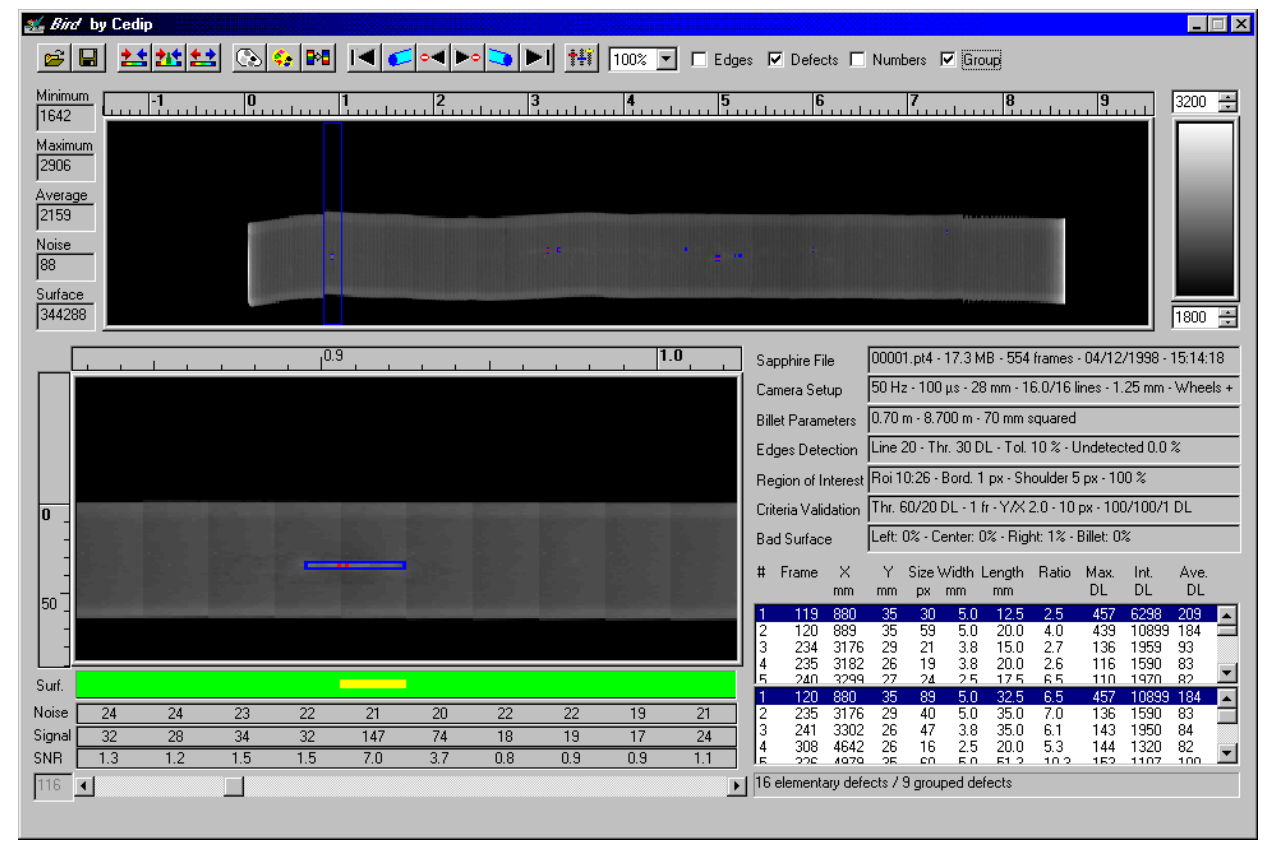

figure 6: IRUS inspection results of a billet. 